EPJ Web of Conferences 49, 17004 (2013)

DOI: $10.1051 /$ epjconf/20134917004

(C) Owned by the authors, published by EDP Sciences, 2013

\title{
Top pair production at ATLAS
}

\author{
Andrey Loginov ${ }^{1, a}$ \\ on behalf of the ATLAS Collaboration \\ ${ }^{1}$ Yale University, Physics Department
}

\begin{abstract}
.
An overview of latest ATLAS measurements of top pair $(t \bar{t})$ production in proton-proton collisions at the LHC at centre-of-mass energies of 7 and $8 \mathrm{TeV}$ is presented. Measurements of the $t \bar{t}$ production cross section $\left(\sigma_{t \bar{t}}\right)$ in various decay channels, including analyses of differential $\sigma_{t \bar{t}}$ distributions and a study of jet multiplicity in $t \bar{t}$ production, as well as searches for $t \bar{t}$ resonances using boosted top techniques and standard methods, are discussed.
\end{abstract}

\section{Introduction}

To date, in $p p$ collision data at the center-of-mass energies of $\sqrt{s}=7 \mathrm{TeV}$ and $\sqrt{s}=8 \mathrm{TeV}$, the CERN Large Hadron Collider (LHC) has successfully delivered two orders of magnitude more top quarks than the Fermilab Tevatron Collider. The top-antitop $(t \bar{t})$ cross section $\left(\sigma_{t \bar{t}}\right)$ precision measurements test the Standard Model (SM) [1] theoretical predictions, which nowadays have percent level accuracy [2]. Differential measurements of $t \bar{t}$ production are especially important for discrimination between different Monte Carlo generators, QCD models and parton distribution functions (PDFs). In addition, future top quark studies will become the best way to constrain systematics on $b$-tagging as well as $c / b$-jet energy scales. On top of that, $t \bar{t}$ events are an important background in various Higgs boson analyses as well as beyond the SM searches, and it is therefore crucial to understand this process in detail. New physics $[3,4]$ can affect both the $t \bar{t}$ production and decay, modifying the observed $\sigma_{t \bar{t}}$ differently in different decay channels and/or affecting differential distributions. In this scenario, it is critical to correctly evaluate all the contributions from SM processes, hence the backgrounds are determined using data-driven techniques whenever possible [5]. In these proceedings an overview of latest ATLAS [6] measurements of $t \bar{t}$ production at the LHC at centre-of-mass energies of 7 and $8 \mathrm{TeV}$ is presented.

\section{$2 \sigma_{t \bar{t}}$ in $\tau_{\text {had }}+$ jets channel at $\sqrt{s}=7 \mathrm{TeV}$}

In the $\tau_{\text {had }}+$ jets channel, one of the top quarks $t \rightarrow W b$ decays produce a tau lepton which then decays hadronically, a $b$-quark and a neutrino, and the other top quark decays hadronically. This study [7] provides the first measurement of the top quark pair cross section in this

\footnotetext{
ae-mail: loginov@fnal.gov
}

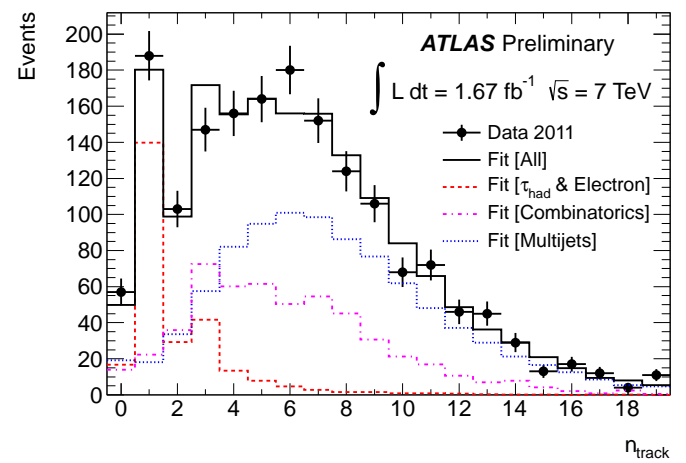

Figure 1: The $n_{\text {track }}$ distribution for $\tau_{\text {had }}$ candidates after all selection cuts [7]. The black circles correspond to data, while the solid histogram is the result of the fit. The red, blue and magenta dashed curves show the fitted contributions from tau and electron "signal", and the multijets and combinatorics backgrounds, respectively.

channel. In addition, the final state is highly relevant for searches beyond the SM. For instance, an enhanced branching fraction in this channel can be a sign of $t \rightarrow$ $b H^{ \pm}\left(H^{ \pm} \rightarrow \tau^{ \pm} v\right)[8]$

Trigger requirements for the analysis are $\geq 4$ jets with $E_{\mathrm{T}}>10 \mathrm{GeV}$ ( $\geq 2$ should be identified as $b$-jets by the High Level Trigger). The offline event selection requires $\geq 5$ jets, including $\geq 2 b$-jets selected with a tagger operating at $60 \%$ efficiency (which corresponds to light flavor jets rejection of 340), and missing transverse momentum $\left(E_{\mathrm{T}}^{\text {miss }}\right)$ satisfying $E_{\mathrm{T}}^{\text {miss }} /\left(0.5 \times \sqrt{\Sigma E_{\mathrm{T}}}\right)>8$, where $\Sigma E_{\mathrm{T}}$ is is the scalar sum of the transverse energy of all objects entering the $E_{\mathrm{T}}^{\mathrm{miss}}$ calculation; events with electrons and muons are excluded from the analysis. The 3 jets (including the $b$-jet with the highest $b$-tagging weight), that give the highest $j j b$ transverse momentum $\left(p_{\mathrm{T}}\right)$ are identified as coming 
Table 1: Systematic uncertainties (\%) on the inclusive $\sigma_{t \bar{t}}$ measurement at $\sqrt{s}=8 \mathrm{TeV}$ in the lepton+jets channel [9].

\begin{tabular}{l|ccc}
\hline Source & $e+\geq 3$ jets & $\mu+\geq 3$ jets & combined \\
\hline Jet/MET reconstruction, calibration & $6.7,-6.3$ & $5.4,-4.6$ & $5.9,-5.2$ \\
Lepton trigger, identification and reconstruction & $2.4,-2.7$ & $4.7,-4.2$ & $2.7,-2.8$ \\
Background normalization and composition & $1.9,-2.2$ & $1.6,-1.5$ & $1.8,-1.9$ \\
$b$-tagging efficiency & $1.7,-1.3$ & $1.9,-1.1$ & $1.8,-1.2$ \\
MC modelling of the signal & \pm 12 & \pm 11 & \pm 11 \\
\hline Total & \pm 14 & \pm 13 & \pm 13 \\
\hline
\end{tabular}

from $t \rightarrow W b \rightarrow j j b$. The remaining non-b highest $p_{\mathrm{T}}$ jet with $p_{\mathrm{T}}>40 \mathrm{GeV}$ and $|\eta|<2.5$ is the hadronic $\tau$ candidate. For the $\tau_{\text {had }}$ candidate a variable $n_{\text {track }}=n_{1}+n_{2}$ is defined, where $n_{1}$ is the number of 'inner' tracks with $\Delta \mathrm{R}\left(\right.$ track, $\left.\tau_{\text {had }}\right)<0.2$, track $p_{\mathrm{T}}>1 \mathrm{GeV}$; and $n_{2}$ is the number of 'outer' tracks in $0.2<\Delta \mathrm{R}\left(\right.$ track, $\left.\tau_{\text {had }}\right)<0.6$, track $p_{\mathrm{T}}>0.5 \mathrm{GeV}$. This variable provides decent separation between a real $\tau_{\text {had }}$ and multijet background.

To extract the signal from the $n_{\text {track }}$ distribution, the data sample is fitted with three templates, as shown in Fig. 1: a tau and electron template (real electrons from $t \bar{t}$ events, either prompt or from leptonic tau decays, contribute significantly to the signal region); a multijets template (gluon-initiated fake taus from multijets); and a combinatorics template (quark-initiated fake taus from ttbar). Then a $\tau /(\tau+e)$ ratio correction is done using Monte Carlo simulation, and the resulting cross section is $\sigma_{t \bar{t}}=$ $200 \pm 19$ (stat) \pm 43 (syst) pb. Dominant systematics for the analysis are initial and final state radiation, ISR/FSR $(12 \%)$ and $b$-jet tagging (10\%) uncertainties.

\section{$3 \sigma_{t \bar{t}}$ in lepton + jets channel at $\sqrt{s}=8 \mathrm{TeV}$}

This analysis [9] is based on the lepton + jets final state, where one of the $\mathrm{W}$ bosons decays leptonically ( $W \rightarrow e v$ or $W \rightarrow \mu v$ ), while the other decays hadronically ( $W \rightarrow$ $j j$ ). The selection criteria are similar to those used for the $\sigma_{t \bar{t}}$ measurement at $7 \mathrm{TeV}$ with a few improvements to cope with harsher pile-up conditions. For example, the lepton $p_{\mathrm{T}}$ cut is raised to $40 \mathrm{GeV}$, and the jet vertex fraction (a variable that quantifies the fraction of track transverse momentum associated to a jet that comes from the hard-scattering interaction) $>0.5$ requirement is added to suppress jets originating from pile-up collisions.

A multivariate likelihood method is used to provide signal-to-background discrimination using lepton pseudorapidity and transformed event aplanarity, $A^{\prime}=\exp (-8 A)$, where $A$ is event aplanarity, as shown in Fig. 2. The number of $t \bar{t}$ events in data is determined from a fit to a distribution of the multivariate likelihood discriminant in data. The resulting cross section is $\sigma_{t \bar{t}}=241 \pm 2$ (stat.) \pm 31 (syst.) \pm 9 (lumi.) pb is in good agreement with theoretical predictions, $\sigma_{t \bar{t}}^{\text {theory }}=238_{-24}^{+22} \mathrm{pb}$. The dominant systematic uncertainty is modelling of the $t \bar{t}$ signal in Monte Carlo as shown in Table 1.
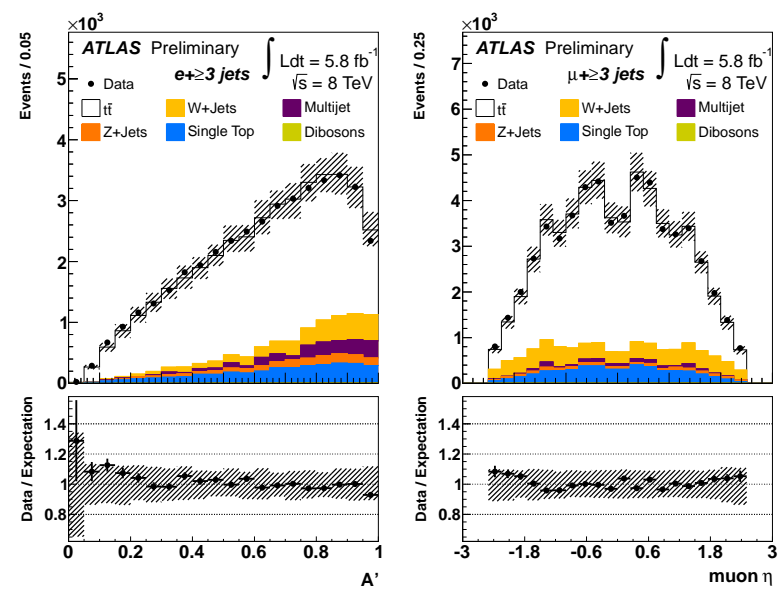

(a) Transformed aplanarity, $e+$ jets

(b) Muon pseudorapidity, $\mu+$ jets

Figure 2: (a) Transformed aplanarity $A^{\prime}=\exp (-8 A)$ (where $A$ is aplanarity) distribution in the $e+$ jets channel; (b) Muon pseudorapidity ( $\eta$ ) distribution in the $\mu+$ jets channel. The distributions in the data (dots) are compared to the model expectations, which include both signal and background processes [9]. The hatched bands display the combined statistical and systematic uncertainties.

\section{Differential $\sigma_{t \bar{t}}$ in the lepton + jets channel at $\sqrt{s}=7 \mathrm{TeV}$}

Differential cross section measurements allow for a test of QCD calculations (and a test of the Standard Model). The analysis [10] focuses on three observables of the $t \bar{t}$ system: the invariant mass $\left(m_{t \bar{t}}\right)$, the transverse momentum $\left(p_{\mathrm{T}, t \bar{t}}\right)$ and the rapidity $\left(y_{t \bar{t}}\right)$. Events are selected in the lepton (electron or muon) + jets channel, requiring 1 high $-p_{\mathrm{T}} e$ or $\mu$, at least 4 high- $p_{\mathrm{T}}$ jets (at least one should be $b$-tagged), large $E_{\mathrm{T}}^{\text {miss }}$ and transverse mass of the lepton- $E_{\mathrm{T}}^{\text {miss }}$ system.

Events consistent with the top pair hypothesis, as defined by a kinematic likelihood fit of the $t \bar{t}$ system, are used. The differential cross sections are normalized to the total $t \bar{t}$ production cross section. To enable direct comparisons to theoretical models, the differential distributions are unfolded to account for detector effects and corrected for acceptance effects as shown in Fig. 3. Bin sizes are optimized to be as small as possible without substantially increasing the total uncertainty after unfolding. The uncertainties of the measurements are dominated by systematics, which are propagated using pseudo-experiments. 


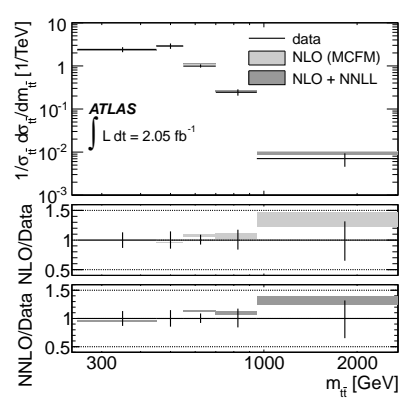

(a)

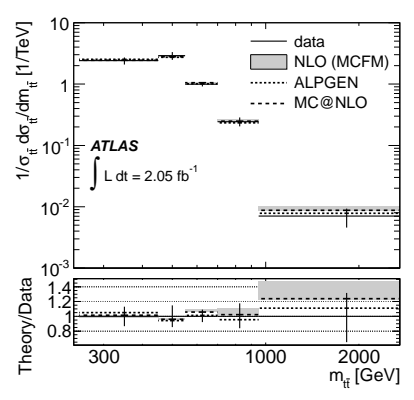

(b)

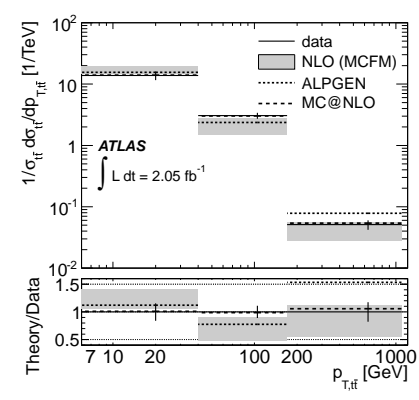

(c)

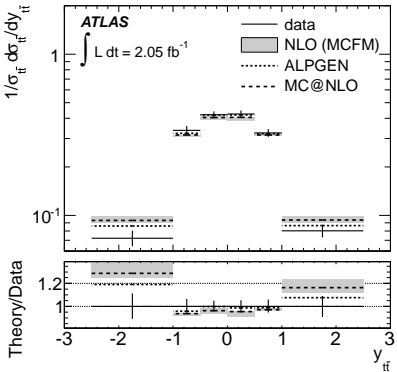

(d)

Figure 3: Relative differential cross section versus (a-b) $m_{t \bar{t}}$, (c) $p_{\mathrm{T}, t \bar{t}}$ and (d) $y_{t \bar{t}}[10]$. The relative cross section is compared to the NLO prediction from MCFM [11]. For $m_{t \bar{t}}$ the results are also compared with the NLO+NNLL prediction [12]. The measured uncertainty represents $68 \%$ confidence level including both statistical and systematic uncertainties. The bands represent theory uncertainties.

Jet-related uncertainties are dominant for $m_{t \bar{t}}$ and $p_{\mathrm{T}, t \bar{t}}$, whereas for $y_{t \bar{t}}$ the dominant contributions are from fake leptons and FSR in addition to the jet uncertainties.

\section{Jet multilplicity in $t \bar{t}$ events}

A measurement of $t \bar{t}$ production with additional jets, as a function of the jet transverse momentum, is important for constraining models of initial and final state radiation at the scale of the top quark mass, as well as to provide a test of perturbative QCD in the LHC energy regime. The analysis [13] is done in the lepton + jets channel with the event selection similar to the one outlined in Sect. 4.

Jet multiplicity measurements are then performed for four jet $p_{\mathrm{T}}$ thresholds $(25,40,60$, and $80 \mathrm{GeV})$ after correction for detector efficiencies, resolution effects and biases, through unfolding, as shown in Fig. 4. The measurements are presented within a kinematic range corresponding to the acceptance of the reconstruction-level event selection. The uncertainty of the measurements is dominated by systematics in all regions. Systematic uncertainties are propagated through unfolding using pseudo-experiments. The unfolded data distributions are compared to various Monte Carlo simulation models.

ISR/FSR variations are implemented by changing the $\alpha_{s}$ (setting the ktfac parameter to 0.5 and 2.0) within the ALPGEN matrix element calculation, while keeping the $\alpha_{s}$ used for the PDF and parton shower fixed. The variations are referred to as $\alpha_{s}$-up (ktfac 0.5) and $\alpha_{s}$-down (ktfac 2.0) variations. Based on this comparison, MC@NLO + HERWIG as well as ALGPEN+PYTHIA with $\alpha_{s}$-up variation are disfavored by data as shown in Fig. 4.

\section{$6 t \bar{t}$ resonances}

The analyses in this section present the results of a search for resonant production of heavy new particles decaying to top quark pairs in lepton + jets and all-hadronic channels. Many new physics scenarios predict the existence of heavy particles that decay to top quark pairs. Limits are set for
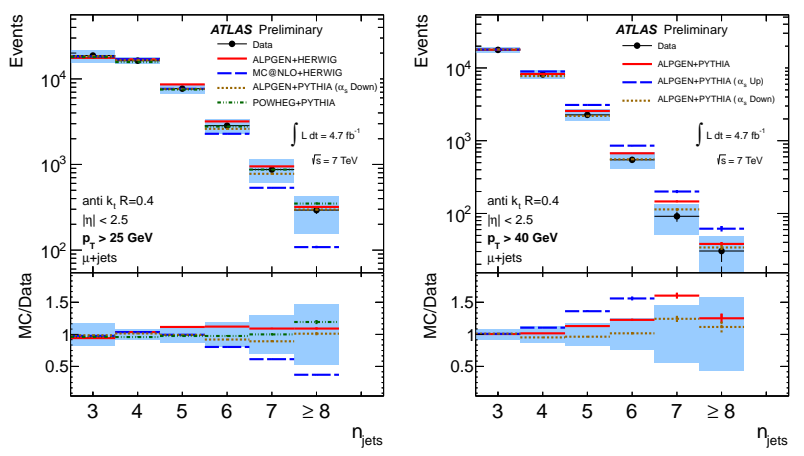

(a) Muon channel, jet $p_{\mathrm{T}}>25 \mathrm{GeV}$ (b) Muon channel, jet $p_{\mathrm{T}}>40 \mathrm{GeV}$

Figure 4: The particle-jet multiplicities [13] for the muon channel and the jet $p_{\mathrm{T}}$ thresholds (a) 25, (b) 40 $\mathrm{GeV}$. The data are shown in comparison with (a) ALPGEN+HERWIG, ALPGEN+PYTHIA $\left(\alpha_{s}\right.$-down variation), MC@NLO+HERWIG and POWHEG+PYTHIA Monte Carlo models, and (b) ALPGEN+PYTHIA and ALPGEN+PYTHIA $\alpha_{s}$-up and $\alpha_{s}$-down variations. The data points are shown in black, whereas the total uncertainty (syst. + stat.) is shown as a shaded band. The MC predictions are shown with their statistical uncertainty.

two distinct classes of models, namely for $Z^{\prime}$ bosons (narrow $t \bar{t}$ resonances) [14] and for Kaluza-Klein gluons (wide $t \bar{t}$ resonances) [15]. The boosted reconstruction technique employed in the analyses is especially important for high invariant mass $t \bar{t}$ resonances as the top quark decay products become more collimated and start to merge.

\subsection{Lepton + jets channel}

This search uses a combination of resolved and boosted reconstruction schemes. In the resolved reconstruction, the standard ATLAS lepton+jets event selection is used. For the boosted reconstruction sample, the three narrow jets from the hadronic top quark decay are expected to merge into one 'fat' jet. Therefore, the data sample used for 


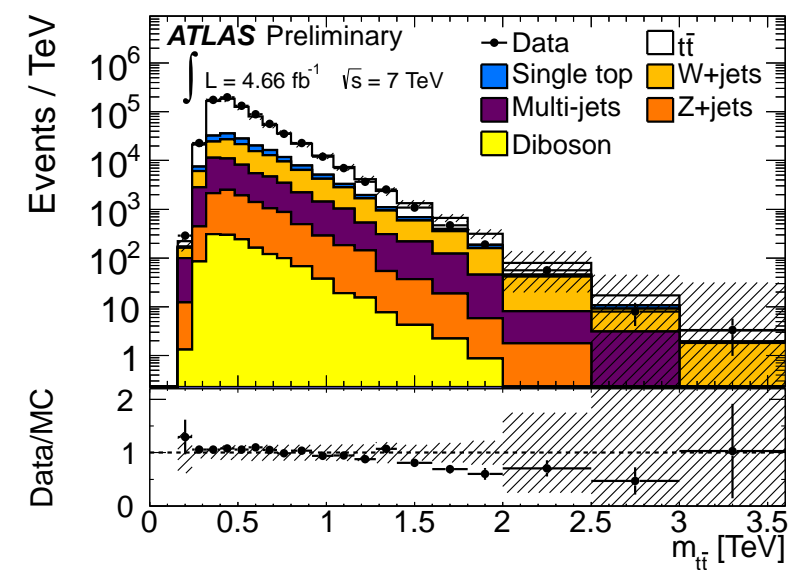

Figure 5: Comparison of the data and the Standard Model prediction for the $t \bar{t}$ invariant mass for combined resolved and boosted selections in the summed $e+$ jets and $\mu+$ jets channels [22]. The shaded areas indicate the total systematic uncertainties

boosted reconstruction was collected with a single largeradius $(\mathrm{R}=1.0)$ jet trigger with transverse momentum threshold of $240 \mathrm{GeV}$. Offline selection requires one high$p_{\mathrm{T}}$ anti- $k_{t}$ [21] jet with $\Delta \mathrm{R}=1.0, p_{\mathrm{T}}>350 \mathrm{GeV}$ and a mass larger than $100 \mathrm{GeV}$. Additionally, because the angular distance between the charged lepton and the $b$-quark decreases as the top quark is boosted more, an isolation requirement with shrinking cone size ('mini-isolation') is used for leptons [22]. The resolved and boosted selections are kept exclusive so that limits can be set on the combined result. Selected data events and expected background yields after the resolved or boosted selections are summarized in Table 2.

Figure 5 shows the resulting $m_{t \bar{t}}$ distributions for the combined resolved and boosted selections for the sum of electron and muon channels. The dominant systematic uncertainty comes from the jet energy scale. The existence of a narrow leptophobic topcolor $Z^{\prime}$ in the range $0.5 \mathrm{TeV}$ $<m_{Z^{\prime}}<1.7 \mathrm{TeV}$ is excluded at $95 \%$ Confidence Level (CL). A wide Kaluza-Klein (KK) gluon for masses between $0.7 \mathrm{TeV}$ and $1.9 \mathrm{TeV}$ is also excluded at 95\% CL.

Table 2: Selected data events and expected background yields after the resolved or boosted selections [22]. The uncertainties on the normalization of the expected background yield are listed.

\begin{tabular}{lcc}
\hline Type & Resolved selection & Boosted selection \\
\hline$t \bar{t}$ & $44113 \pm 4720$ & $917 \pm 98$ \\
\hline Single top & $3243 \pm 250$ & $49 \pm 4$ \\
\hline Multi.jets & $3321 \pm 1660$ & $30 \pm 15$ \\
\hline$W+$ jets & $2359 \pm 472$ & $46 \pm 9$ \\
\hline$Z+$ jets & $539 \pm 259$ & $8.5 \pm 4.1$ \\
\hline Di-bosons & $124 \pm 43$ & $0.86 \pm 0.29$ \\
\hline Total & $57978 \pm 5170$ & $1118 \pm 101$ \\
\hline Data & 61573 & 1080 \\
\hline
\end{tabular}

\subsection{All-hadronic channel}

In this analysis [23] the final states with high- $p_{\mathrm{T}}$ top quarks that decay hadronically and where the decay products are collimated in the direction of the top-quark are considered. Such searches require the top quarks to have $p_{\mathrm{T}}$ in excess of 200-300 GeV and require rejection of the large background of gluon jets, light-quark jets, as well as $c$ - and $b$-jets. The all-hadronic signature studied in the analysis is complementary to the one presented in Sect. 6.1. Two complementary algorithms are used in the analysis to identify top-quark decays and reconstruct the top-quark momentum.

The first method is the HEPTopTagger algorithm [16, 17] that tests the substructure of a jet reconstructed with the Cambridge / Aachen (C/A) algorithm [18] with $\Delta \mathrm{R}=$ 1.5 for its compatibility with a hadronic top quark decay. This method is efficient for jets with $p_{\mathrm{T}}>200 \mathrm{GeV}$, and it works by forming subjets in the bigger $\Delta \mathrm{R}=1.5$ fat jet, removing soft contamination, and then reconstructing $t \rightarrow W b \rightarrow b q q$ decay products and testing compatibility of subjets with the $\mathrm{W}$ boson and top quark decays. The candidate top jet mass cut used for the method is 140-210 GeV.

The second method is the Top Template Tagger algorithm $[19,20]$ that compares templates of boosted top quark decays to the observed energy deposits to find the best match. The Top Template Tagger uses jets reconstructed with the anti- $k_{t}$ algorithm with a distance parameter of $\Delta \mathrm{R}=1.0$ and is optimised to identify top quarks with $p_{\mathrm{T}}>500 \mathrm{GeV}$. The candidate top jet mass cut used for the method is $122-222 \mathrm{GeV}$.

Table 3: Expected (Exp.) and observed (Obs.) exclusion regions on the leptophobic $Z^{\prime}$ boson and Randall-Sundrum KK gluon masses [23].

\begin{tabular}{l|c|c}
\hline Model & Obs. Limit $(\mathrm{TeV})$ & Exp. Limit $(\mathrm{TeV})$ \\
\hline \multicolumn{3}{|c}{ HEPTopTagger } \\
\hline$Z^{\prime}$ & $0.70<m_{Z^{\prime}}<1.00$ & $0.68<m_{Z^{\prime}}<1.16$ \\
& $1.28<m_{Z^{\prime}}<1.32$ & \\
KK gluon & $0.70<m_{g_{K K}}<1.48$ & $0.70<m_{g_{K K}}<1.52$ \\
\hline \multicolumn{3}{|c}{ Top Template Tagger } \\
\hline KK gluon & $1.02<m_{g_{K K}}<1.62$ & $1.08<m_{g_{K K}}<1.62$ \\
\hline \multicolumn{3}{c}{ Combined $($ Observed Limit $)$} \\
\hline$Z^{\prime}$ & $0.70<m_{Z^{\prime}}<1.00,1.28<m_{Z^{\prime}}<1.32$ \\
KK gluon & $0.70<m_{g_{K K}}<1.62$ \\
\hline
\end{tabular}

For the HEPTopTagger, to avoid relying on a particular topology, the trigger is a) at least 1 jet with $E_{\mathrm{T}}>100 \mathrm{GeV}$ and large $\Sigma E_{\mathrm{T}} \mathrm{OR}$ b) at least 5 jets with $E_{\mathrm{T}}>50 \mathrm{GeV}$. For the Top Template Tagger, the events are selected by the trigger that requires one anti- $k_{t} \Delta \mathrm{R}=1.0$ jet with $E_{\mathrm{T}}>$ $240 \mathrm{GeV}$. For the event selection, at least two fat jets are required for both algorithms. For the HEPTopTagger the jet $p_{\mathrm{T}}$ is required to be greater than $200 \mathrm{GeV}$, while for the Top Template Tagger the leading (subleading) jet $p_{\mathrm{T}}$ is required to be greater than 500 (450) GeV. Additionally, at least two $b$-tagged anti- $k_{t} \Delta \mathrm{R}=0.4$ jets within 

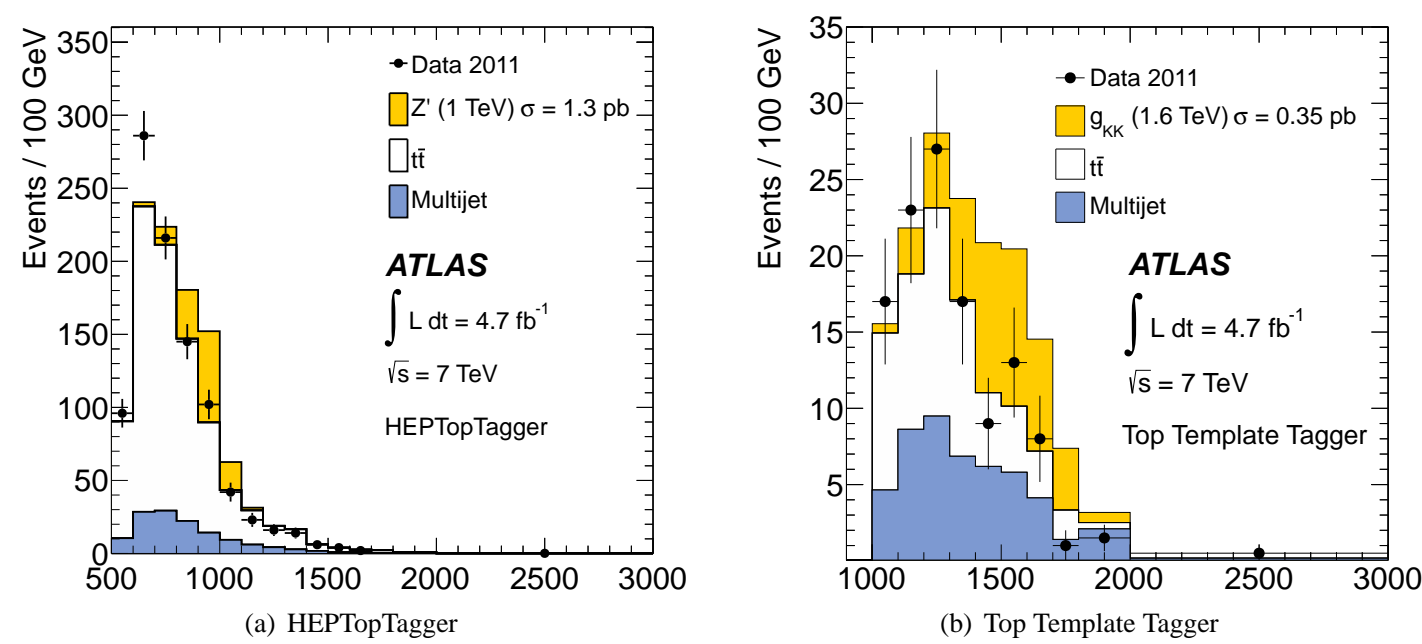

(b) Top Template Tagger

Figure 6: Distributions of the $t \bar{t}$ invariant mass $m_{t \bar{t}}$ for the HEPTopTagger and the Top Template Tagger data [23].

$\Delta \mathrm{R}=1.4(0.4)$ around the axis of a fat jet are required for HEPTopTagger (Top Template Tagger) analysis.

The results obtained with the two methods are shown in Fig. 6 together with a hypothetical $Z^{\prime}$ signal with $m_{Z^{\prime}}=$ $1 \mathrm{TeV}$ (a) and a hypothetical KK gluon signal with $m_{K K g}=$ $1.6 \mathrm{TeV}$ (b). To combine the limits from these two analyses, the results from the tagger with the lower expected exclusion limit are selected, as summarized in Table 3.

\section{Summary}

To summarize, LHC keeps providing top quarks in unprecedented quantities, which allows detailed precision measurements and detailed studies. Top quark physics at LHC is a crucial milestone in both measuring the properties of the top quark, the heaviest particle discovered so far, and in searches for heavy mass physics beyond the Standard Model, to which the top quark pair production process is often an important background.

\section{References}

[1] S.L. Glashow, Nucl. Phys. 22 588, (1961); S. Weinberg, Phys. Rev. Lett. 19 1264, (1967); A. Salam, Proc. 8th Nobel Symposium, Stockholm, (1979).

[2] M. Aliev, H. Lacker, U. Langenfeld, S. Moch, P. Uwer and M. Wiedermann, Comput. Phys. Commun. 182, 1034 (2011) arXiv:1007.1327 [hep-ph].

[3] For instance, a $t^{\prime}$ decaying in $W q$ would mimic the $t \bar{t}$ lepton+jets signature. J. Silva-Marcos, J. High Energy Phys. 0212, 036 (2002); S. Sultansoy et al., Acta Phys. Polon. B37, 2839 (2006).

[4] J. R. Incandela, A. Quadt, W. Wagner, D. Wicke, Prog.Part.Nucl.Phys. 63,239-292,2009; arXiv:0904.2499v3 [hep-ex].

[5] A. Loginov, Nuovo Cim. C 033N4, 175 (2010), ATL-PHYS-PROC-2010-067.
[6] ATLAS Collaboration, JINST 3, S08003 (2008).

[7] ATLAS Collaboration, ATLAS-CONF-2012-032.

[8] ATLAS Collaboration, ATLAS-CONF-2012-011.

[9] ATLAS Collaboration, ATLAS-CONF-2012-149.

[10] ATLAS Collaboration, arXiv:1207.5644 [hep-ex], submitted to Eur. J. Phys. C.

[11] J. Campbell, K. Ellis, C. Williams. http://mcfm.fnal.gov/.

[12] V. Ahrens, A. Ferroglia, M. Neubert, B. D. Pecjak, and L. L. Yang, JHEP 1009 (2010) 097, arXiv:1003.5827 [hep-ph].

[13] ATLAS Collaboration, ATLAS-CONF-2012-155.

[14] R. M. Harris, C. T. Hill and S. J. Parke, hep-ph/9911288.

[15] K. Agashe, A. Belyaev, T. Krupovnickas, G. Perez and J. Virzi, Phys. Rev. D 77, 015003 (2008), hep-ph/0612015; B. Lillie, L. Randall and L. -T. Wang, JHEP 0709, 074 (2007), hep-ph/0701166.

[16] T. Plehn, G. P. Salam, and M. Spannowsky, Phys. Rev. Lett. 104 (Mar, 2010) 111801, arXiv:0910.5472.

[17] T. Plehn, M. Spannowsky, M. Takeuchi, and D. Zerwas, JHEP 1010 (2010) 078, arXiv:1006.2833.

[18] Y. Dokshitzer, G. Leder, S. Moretti, and B. Webber, JHEP 9708 (1997) 001, hep-ph/9707323.

[19] L. G. Almeida, S. J. Lee, G. Perez, G. Sterman, and I. Sung, Phys. Rev. D 82 (Sep, 2010) 054034, arXiv:1006.2035.

[20] L. G. Almeida, O. Erdogan, J. Juknevich, S. J. Lee, G. Perez, and G. Sterman, Phys.Rev. D 85 (2012) 114046, arXiv:1112.1957.

[21] M. Cacciari, G. P. Salam and G. Soyez, JHEP 0804, 063 (2008), arXiv:0802.1189 [hep-ph].

[22] ATLAS Collaboration, ATLAS-CONF-2012-136.

[23] ATLAS Collaboration, JHEP 1301, 116 (2013), arXiv:1211.2202 [hep-ex]. 